\title{
Spectroscopic observations of the quiescent neutron star system 4U 2129+47 (=V1727 Cygni) (Research Note)
}

\author{
M. S. Bothwell ${ }^{1,2}$, M. A. P. Torres ${ }^{3}$, M. R. Garcia ${ }^{3}$, and P. A. Charles ${ }^{4}$ \\ 1 Institute of Astronomy, University of Cambridge, Madingley Road, Cambridge CB3 OHA, UK \\ e-mail: bothwell@ast. cam.ac.uk \\ 2 School of Physics and Astronomy, Southampton University, Southampton SO17 1BJ, UK \\ ${ }^{3}$ Harvard-Smithsonian Center for Astrophysics, Cambridge, MA 02138, USA \\ 4 South African Astronomical Observatory, PO Box 9, Observatory, 7935, South Africa
}

Received 5 February 2008 / Accepted 20 March 2008

ABSTRACT

Context. In quiescence, the proposed optical counterpart to the neutron star, low mass X-ray binary 4U 2129+47 (V1727 Cyg) shows a spectrum consistent with a late F-type subgiant and no radial velocity variations on the $5.24 \mathrm{~h}$ binary period. This could imply that V1727 Cyg is a chance line of sight interloper. Radial velocity measurements, however, showed evidence for a longer term $\sim 40 \mathrm{~km} \mathrm{~s}^{-1}$ shift, which suggested that $4 \mathrm{U} 2129+47$ could be a hierarchical triple system, with the F-type star in a wide orbit about the inner low mass X-ray binary.

Aims. In order to confirm the long-term radial velocity shift reported in Garcia et al. (1989, ApJ, 341, L75) and its amplitude, we obtained spectroscopic observations of V1727 Cyg during 1996 and 1998 with the William Herschel Telescope using the ISIS spectrograph.

Methods. We determined radial velocities from the ISIS spectra by means of the cross-correlation technique with a template spectrum. Results. The resulting radial velocities show variations with a maximum amplitude of $\sim 40 \mathrm{~km} \mathrm{~s}^{-1}$, confirming previous results and supporting the F-type star as being the third body in a hierarchical triple system. The odds that this star could be an interloper are $\sim 3 \times 10^{-6}$.

Key words. X-rays: binaries - techniques: radial velocities - stars: individual: V1727 Cygni

\section{Introduction}

4U 2129+47 was discovered as an active, but weak X-ray source in the fourth UHURU survey (Forman et al. 1978), and its optical counterpart was identified as V1727 Cyg (Thorstensen et al. 1979). V1727 Cyg was found to be of $\sim 17$ th mag, and exhibited a large amplitude $(\Delta B \sim 1.5)$ photometric periodicity of $5.24 \mathrm{~h}$. This was taken to be indicative of the orbital period, with the photometric modulation ascribed to X-ray heating of the companion (Thorstensen et al. 1979). Analysis of the X-ray light curve led to the development of a model where the system is viewed edge on and surrounded by an accretion disc corona (White \& Holt 1982). Attempts to measure the mass of the compact object produced confusing results, with radial velocity studies implying a compact object mass of $0.6 \pm 0.2 M_{\odot}$ (Horne et al. 1986). This led to the conclusion that the binary system was a cataclysmic variable. However, the discovery of an X-ray burst confirmed the identity as a neutron star X-ray binary (Garcia \& Grindlay 1987). Assuming a neutron star primary and a $5.24 \mathrm{~h}$ period, the empirical relationship described by Robinson (1976) between the companion mass and the binary period indicates a mass of $0.59 \leq M\left(M_{\odot}\right) \leq 0.80$ and a radius of $0.59 \leq R\left(R_{\odot}\right) \leq 0.68$ (Thorstensen et al. 1979). These parameters suggest a K-M V spectral type for the companion star.

X-ray observations in 1983 failed to detect 4U 2129+47, and contemporaneous optical observations showed that V1727 Cyg had dimmed to $\sim 18.5$ th mag (Pietsch et al. 1986). This period of quiescence was a good opportunity for detecting the companion star with a view to constraining the orbital parameters of the system. The first such study was conducted by Garcia et al. (1989). Spectra were taken during four observing runs between June 1987 and October 1988 in order to obtain more accurate measurements of the neutron star mass without the strong influence of accretion disc emission. The results were surprising: no variations on a $5.24 \mathrm{~h}$ period were detected. From the upper limits on the orbital radial velocity variations, the neutron star's mass appeared to be $<0.1 M_{\odot}$ (Garcia et al. 1989), which is obviously inconsistent with a neutron star primary. Moreover, the June 1987 run provided a mean velocity significantly higher (by $\sim 40 \mathrm{~km} \mathrm{~s}^{-1}$ ) than observed in the other nights. Another surprise was the identification of V1727 Cyg as an F7-IV star - a stable $5.24 \mathrm{~h}$ orbit about a neutron star is smaller than the radius of an F7-IV star (by a factor of $\sim 1.5$ ). These results have led to the suggestion that $4 \mathrm{U} 2129+47$ is a hierarchical triple system. The observed F-type star is postulated to be in a wide orbit about the centre of mass of the close pair, while the inner companion is perhaps a K-type dwarf (Garcia et al. 1989). The F7 star dominates the optical spectrum in the current quiescent state, and so radial velocity shifts on a $5.24 \mathrm{~h}$ period are not observed. Garcia et al. (1989) suggested that a 30 day outer period could account for the on-off cycles seen in $4 \mathrm{U} 2129+47$. An outer period on this order would drive a periodic eccentricity in the inner binary on a 45 year timescale, consistent with the on and off states that have been observed since the 1930s. The radial velocity data 
previously obtained and newly reported herein are consistent with such a period. Chevalier et al. (1989) have pointed out that even without the observed radial velocity shift, the lack of large radial velocity variations would lead to the postulation of a third stellar component.

An obvious alternative to the triple hypothesis is a chance line of sight alignment. Ground based studies show that the on and off state optical counterparts are coincident to $0.26^{\prime \prime}$ indicating that the likelihood of a chance alignment is $10^{-3}$ (Thorstensen et al. 1988). HST imaging shows that there is no nearby companion of comparable magnitude within 0.04 " (Deutsch et al. 1996). Scaling this radius to the ground based results further reduces the chance alignment probability to $2 \times 10^{-5}$.

In this paper, we present spectra of V1727 Cyg taken in order to analyse its systemic radial velocity over a time interval similar to the long ( $\sim 30$ day) period predicted for the late F-type star if this is the outer component of a triple system (Garcia et al. 1989). Previous radial velocity data were acquired during short observing runs (over at most 2 nights), separated by periods of many months. In order to test the proposed $\sim 30$ day period it was necessary to conduct a study over a time interval of $\sim 1$ month, with data points separated by about a week.

\section{Observations}

Optical spectra were acquired in service mode during 1996 and 1998 using the $4.2 \mathrm{~m}$ William Herschel Telescope on La Palma equipped with the dual-beam ISIS spectrograph. V1727 Cyg was observed with both arms of the instrument.

The 1996 data set was acquired on August 5/12/19/25 UT. The observations were made using the R1200R grating and TEK2 CCD in the red arm and the R600B and TEK1 CCD in the blue arm, giving a dispersion of $0.40 \AA \mathrm{pixel}^{-1}$ and $0.78 \AA$ pixel $^{-1}$, respectively. The slit width used ranged from 1 .'0 to 1 '. 3 . From measurements of the arc-lamp and night sky emission lines, we find that this yielded a spectral resolution from 1.0 to $1.2 \AA$ (red arm) and 1.4 to $2.0 \AA$ (blue arm). The red and blue data cover the wavelength range $\lambda \lambda 6370-6760$ and $\lambda \lambda 4340-4910$. On each night, two to three $30 \mathrm{~min}$ spectra of V1727 Cyg were taken, along with spectra of a template star, either BD+47 4219 or HD 222368 (both F7-IV stars). HD 222368 is a GCRV star with a velocity of $5.0 \pm 0.9 \mathrm{~km} \mathrm{~s}^{-1}$. In total, 11 spectra of V1727 Cyg were obtained. Calibration frames were also acquired, in particular arc lamp spectra. However, the night of August 4 had no flat fields taken. The data have been included in the analysis as the signal to noise was high enough to mask the fixed pattern noise: performing a cross correlation between an August 5 object spectra and an identically extracted strip from a flat field produced no peak, indicating that the fixed pattern noise is small. The night of August 12 (with 2 target spectra) was discarded, as the arc lamp spectra were not rigorously taken close in time to the target spectrum, leading to an uncertain wavelength calibration.

The 1998 data set consists of 6 nights of data: June 19/27, July $3 / 17 / 22$ and August 2 UT. The observations were taken with the R1200R grating and TEK2 CCD on the red arm and the R1200B grating and EEV10 CCD on the blue arm of the spectrograph. The slit width used ranged from $11^{\prime \prime} 0$ to 1 .' 2 . In the red arm, this provided a spectral resolution from 0.8 to $1.5 \AA$. The blue arm data was of poor quality and were not used in the analysis. The red data covered the wavelength interval $\lambda \lambda 6310-6710$. During each night two to three spectra of V1727 Cyg with

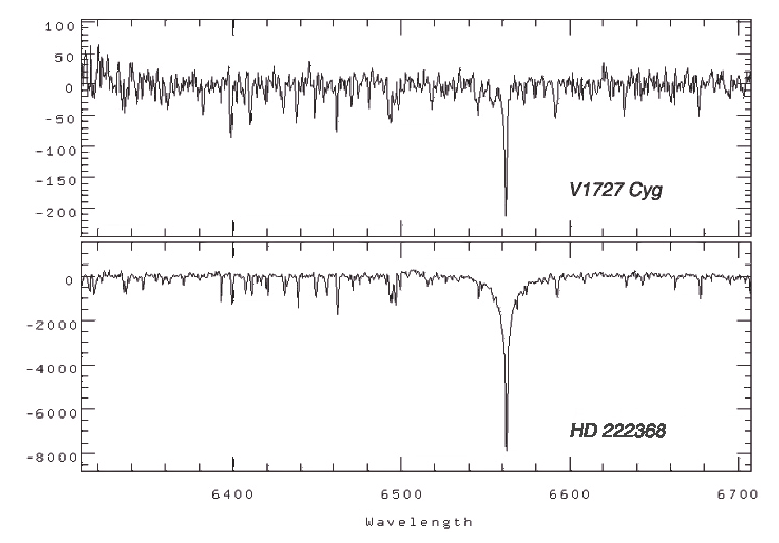

Fig. 1. Summed spectra of V1727 Cyg compared to that of the template spectrum used, HD 222368.

integration times ranging from 15 to 30 min were taken, along with all associated calibration frames. Spectra of BD+47 4219 were also acquired each night (except on July 3). A total of 15 spectra of V1727 Cyg were taken. The two spectra acquired on June 27 were discarded due to their low quality. Furthermore, the night of July 16 was unsuitable for use due to instrumental problems.

Both data sets were reduced with standard IRAF routines and the spectra were extracted with the IRAF KPNOSLIT package.

\section{Analysis}

Velocities were computed using the Fourier cross-correlation technique developed by Tonry \& Davis (1979) and implemented in the IRAF task FXCOR. A sum of three HD 222368 spectra (taken on 1996 August 18 when conditions were very good) was used as a template spectrum. Figure 1 shows this composite spectrum from the red arm along with a sum of four V1727 Cyg spectra. Prior to the cross-correlation, the target and template spectra were re-sampled into a common logarithmic wavelength scale and normalized by dividing with the result of fitting a loworder spline to the continuum. Correlation was performed without $\mathrm{H} \alpha$ in the red, to ensure that any possible residual emission from the accretion disc did not interfere with the measured velocity of V1727 Cyg. In the blue, $\mathrm{H} \beta$ was included, as (besides the G-band) the spectra lack well defined features in the available region, and without $\mathrm{H} \beta$ correlation fits were poor.

Figure 2 and Table 1 show the radial velocity of V1727 Cyg from the 1996 and 1998 data sets. The radial velocities shown in Fig. 2 are weighted averages of radial velocities obtained from the red and (when available) blue spectra. All velocities are heliocentric. Table 1 also shows the average radial velocities obtained as derived using only the blue or ned spectra. As in Garcia et al. (1989) the errors on the individual velocities produced by FXCOR have been computed as $\sigma=C /(1+r)$, where $C$ is a constant determined from the observed scatter in the velocity of comparison stars, and $r$ is the correlation coefficient (see Tonry \& Davis 1979). Gaussian statistics were used throughout when calculating mean values. Checking the wavelength of night sky emission lines served as a zero point calibration for the data: these lines are seen only at wavelengths longer than $\sim 5000 \AA$, so this method could not be used for the blue frames. The average night sky line velocity is approximately $-0.4 \mathrm{~km} \mathrm{~s}^{-1}$, which is consistent with zero given the errors. The wavelength of each night sky line was found by finding the centroid of a Gaussian function fitted to each line. Both the larger error bar for the 


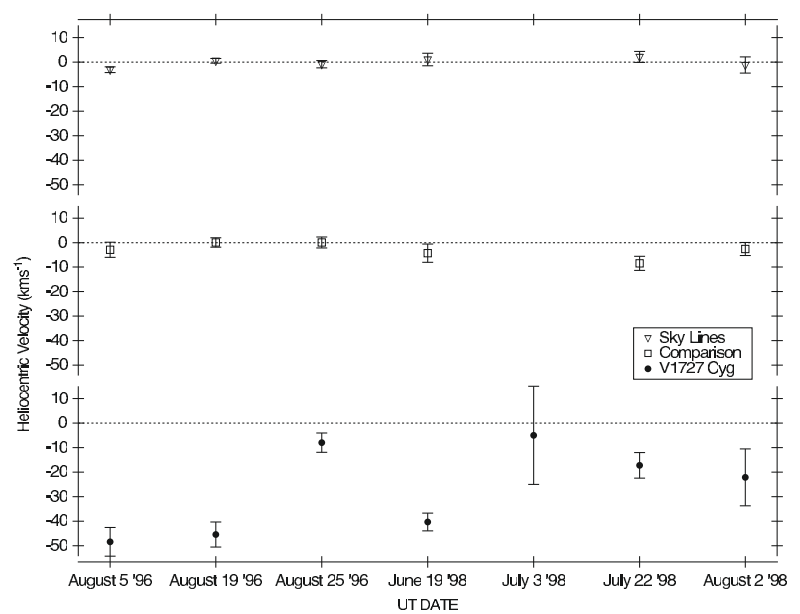

Fig. 2. New radial velocity data for V1727 Cyg (bottom) from 1996 and 1998 (see Table 1). Also plotted are sky line velocities as a zero point check (top plot). These velocities were calculated from the difference between the measured and published (Osterbrock et al. 1996) wavelengths of sky emission lines. Comparison star velocities are shown in the middle plot.

Table 1. Heliocentric radial velocities in $\mathrm{km} \mathrm{s}^{-1}$ ) for V1727 Cyg in quiescence.

\begin{tabular}{cccc}
\hline \hline $\begin{array}{c}\text { Date } \\
\text { (UT) }\end{array}$ & $\begin{array}{c}\text { Radial velocity } \\
\text { (blue and red arm) }\end{array}$ & $\begin{array}{c}\text { Radial velocity } \\
\text { (blue arm) }\end{array}$ & $\begin{array}{c}\text { Radial velocity } \\
\text { (red arm) }\end{array}$ \\
\hline 1996 Aug. 5.0 & $-48.4 \pm 5.8$ & $-48.7 \pm 7.8$ & $-48.3 \pm 2.7$ \\
1996 Aug. 19.2 & $-45.4 \pm 5.1$ & $-48.3 \pm 6.0$ & $-39.3 \pm 3.4$ \\
1996 Aug. 25.1 & $-8.0 \pm 3.9$ & $-3.1 \pm 4.2$ & $-14.4 \pm 4.0$ \\
1998 Jun. 19.2 & $-40.3 \pm 3.6$ & - & $-40.3 \pm 3.6$ \\
1998 Jul. 3.2 & $-5 \pm 20$ & - & $-5 \pm 20$ \\
1998 Jul. 22.1 & $-17.2 \pm 5.2$ & - & $-17.2 \pm 5.2$ \\
1998 Aug. 2.1 & $-22 \pm 11$ & - & $-22 \pm 11$ \\
\hline
\end{tabular}

V1727 Cyg radial velocity and the lack of sky line measurements on 1998 July 3 are due to the fact that the spectra were obtained during dawn. As noted in Sect. 2, no radial velocity templates were observed during this night.

\section{Discussion}

Our data do not show the expected short term, $\sim 300 \mathrm{~km} \mathrm{~s}^{-1}$ semi-amplitude radial velocity variations indicative of a neutron star primary. This is consistent with all previous studies of V1727 Cyg in quiescence (see e.g. Garcia et al. 1989; Garcia 1989; Chevalier et al. 1989; Cowley \& Schmidtke 1990). Furthermore, the longer term velocity variations found by Garcia et al. 1989 are confirmed in this new dataset. The $40 \mathrm{~km} \mathrm{~s}^{-1} \mathrm{am}-$ plitude of the variation is similar to that found earlier, and the variation is detected between 7 and $10 \sigma$ level.

If we accept that the late F-type subgiant is indeed orbiting an unseen companion (or companions) we can ask what are the odds that it could still be a line of sight interloper, rather than physically associated with 4 U 2129+47? The fraction of multiple systems in the Galaxy is an active topic of study, with estimates ranging from $10 \%$ to $80 \%$ (Lada 2006). It is now clear that the fraction depends upon mass, with more massive stars more likely to be members of multiple systems. Also, the majority of the systems are widely detached and show only small (few $\mathrm{km} \mathrm{s}^{-1}$ ) radial velocity variations. We used the study of Duquennoy \& Mayor (1991) to estimate the fraction of F7 stars that would show velocity variations of at least $40 \mathrm{~km} \mathrm{~s}^{-1}$ (corresponding to a radial velocity semi-amplitude $K$ of $20 \mathrm{~km} \mathrm{~s}^{-1}$ ). This study measured orbital velocity variations of a sample of F7 to G8 stars in order to determine orbital periods and multiplicity frequency. The mean period in the sample was $\log (P)=4.8$ with a roughly Gaussian dispersion of $\log (P)=2.3$ with $P$ in days. From Table 2 of Duquennoy \& Mayor (1991) we estimate that $K=20 \mathrm{~km} \mathrm{~s}^{-1}$ corresponds approximately to $P=100$ days. The fraction of systems with periods less than 100 days, and therefore likely to show velocity variation of $40 \mathrm{~km} \mathrm{~s}^{-1}$ or more, is approximately 1 in 7 . Therefore the odds that V1727 Cyg could be an interloper are reduced from $2 \times 10^{-5}$ to approximately $3 \times 10^{-6}$.

Recently, Bozzo et al. (2007) have presented time analysis of two XMM-Newton observations of 4 U 2129+47 in quiescence obtained $\sim 22$ days apart, finding evidence for a delay of $\sim 200$ s for the mid-eclipse times measured from the X-ray observations. This delay can be explained as due to the orbital motion of the compact $4 \mathrm{U} 2129+47$ binary around the center of mass of a triple system. In light of these new observations, coupled with the radial velocity measurements presented in this paper, we conclude that the triple hypothesis best explains all features of $4 \mathrm{U} 2129+47$, and that the late F-type subgiant is the outer component of the triple system.

\section{Conclusions}

The X-ray source $4 \mathrm{U} 2129+47$ has been a candidate hierarchical triple for some time. The optical counterpart, V1727 Cyg, has features inconsistent with those expected for a close binary companion, and previously displayed radial velocity variation over several weeks. The new spectroscopic measurements presented here confirm this long term shift of $\sim 40 \mathrm{~km} \mathrm{~s}^{-1}$, which strongly suggests that $4 \mathrm{U} 2129+47$ is indeed a triple. However, the outer period is yet to be observed directly. Doing so would allow for more sophisticated modeling of the system. It may also help understand the evolutionary history of 4U 2129+47, which as of now is somewhat elusive.

Acknowledgements. This paper makes use of data obtained from the Isaac Newton Group Archive which is maintained as part of the CASU Astronomical Data Centre at the Institute of Astronomy, Cambridge. We acknowledge NASA/ Chandra Data Center Contract NAS8-03060, which provides partial support for MRG.

\section{References}

Bozzo, E., Falanga, M., Papitto, A., et al. 2007, A\&A, 476, 301 Chevalier, C., Ilovaisky, S. A., Motch, C., et al. 1989, A\&A, 217, 108 Cowley, A. P., \& Schmidtke, P. C. 1990, AJ, 99, 678

Deutsch, E. W., Margon, B., Wachter, S., et al. 1996, ApJ, 471, 979 Duquennoy, A., \& Mayor, M. 1991, A\&A, 248, 485

Forman, W., Jones, C., Cominsky, L., et al. 1978, ApJS, 38, 357 Garcia, M. R. 1989, Proc. 23rd ESLAB Symp., ESA SP-296, 151 Garcia, M. R., \& Grindlay, J. E. 1987, ApJ, 313, L59

Garcia, M. R., Bailyn, C. D., Grindlay, J. E., et al. 1989, ApJ, 341, L75 Horne, K., Verbunt, F., \& Schneider, D. P. 1986, MNRAS, 218, 63 Lada, C. J. 2006, ApJ, 640, L63

Osterbrock, D. E., Fulbright, J. P., Martel, A. R., et al. 1996, PASP, 108, 277 Pietsch, W., Steinle, H., Gottwald, M., et al. 1986, AJ, 157, 23

Robinson, E. L. 1976, ApJ, 203, 485

Thorstensen, J., Charles, P., Bowyer, S., et al. 1979, ApJ, 233, L57 Thorstensen, J. R., Brownsberger, K. R., Mook, D. E., et al. 1988, ApJ, 334, 430 Tonry, J., \& Davis, M. 1979, AJ, 84, 1511 White, N., \& Holt, S. 1982, ApJ, 257, 318 